\title{
Erratum to: The prognostic ability of the STarT Back Tool was affected by episode duration
}

\author{
Lars Morso $^{1} \cdot$ Alice Kongsted $^{2,3} \cdot$ Lise Hestbaek $^{2,3} \cdot$ Peter Kent $^{3}$
}

Received: 19 April 2016/Revised: 19 April 2016/Accepted: 19 April 2016/Published online: 9 May 2016

(c) Springer-Verlag Berlin Heidelberg 2016

\section{Erratum to: Eur Spine J (2016) 25:936-944 \\ DOI 10.1007/s00586-015-3915-0}

Unfortunately, there is a mistake in the published version of Table 1. In the last row of the table, the numbers corresponding to the proportion of people in the STarT Back Tool risk subgroups High Risk and Low Risk had been swapped. When these numbers are correctly swapped back, they then correspond with the numbers presented in lines $10-20$ of the results section. This misleading error was only in the table layout and did not affect the results or the conclusions of the paper.

The online version of the original article can be found under doi:10.1007/s00586-015-3915-0.

Lars Morso

lars.morsoe@rsyd.dk

1 Centre for Quality, Region of Southern Denmark, P.V.

Tuxensvej 5, 5500 Middelfart, Denmark

2 Nordic Institute of Chiropractic and Clinical Biomechanics, Odense, Denmark

3 Department of Sports Science and Clinical Biomechanics, University of Southern Denmark, Odense, Denmark 
Table 1 Baseline characteristics of included cohorts

\begin{tabular}{|c|c|c|c|c|c|}
\hline & $\begin{array}{l}\text { Chiropractic cohort } \\
(n=416)\end{array}$ & $\begin{array}{l}\text { GP cohort } \\
(n=244)\end{array}$ & $\begin{array}{l}\text { Physiotherapy cohort } \\
(n=200)\end{array}$ & $\begin{array}{l}\text { Spine centre cohort } \\
(n=960)\end{array}$ & $\begin{array}{l}\text { Test for differences between } \\
\text { cohorts }\end{array}$ \\
\hline \multicolumn{6}{|l|}{ Age in years } \\
\hline Mean $\left(\mathrm{SD}^{\mathrm{a}}\right)$ & $42.9(11.8)$ & $46.1(11.4)$ & $50.5(16.3)$ & $52.0(14.1)$ & Chiro $<$ GP $<$ others \\
\hline Female, proportion & $187(45.0 \%)$ & $130(53.3 \%)$ & $115(57.5 \%)$ & $521(54.3 \%)$ & Chiro $<$ others \\
\hline \multicolumn{6}{|l|}{ Duration in weeks } \\
\hline $0-2$ weeks & $252(62.1 \%)$ & $83(38.1 \%)$ & $44(22.8 \%)$ & $15(1.6 \%)$ & \multirow[t]{4}{*}{ All differ from each other } \\
\hline $2-4$ weeks & $48(11.8 \%)$ & $26(11.9 \%)$ & $33(17.1 \%)$ & $34(3.7 \%)$ & \\
\hline 4-12 weeks & $46(11.3 \%)$ & $33(15.1 \%)$ & $42(21.8 \%)$ & $137(14.7 \%)$ & \\
\hline$>12$ weeks & $60(14.8 \%)$ & $76(34.9 \%)$ & $74(38.3 \%)$ & $746(80.0 \%)$ & \\
\hline \multicolumn{6}{|c|}{ Previous low back pain episodes } \\
\hline Previous episodes & $331(81.5 \%)$ & $190(86.4 \%)$ & $152(79.2 \%)$ & $778(81.8 \%)$ & No differences \\
\hline \multicolumn{6}{|c|}{ Pain intensity ${ }^{\mathrm{b}}(0-10 \text { scale })^{\mathrm{c}}$} \\
\hline $\begin{array}{l}\text { Low back pain, } \\
\text { mean }\left(\mathrm{SD}^{\mathrm{a}}\right)\end{array}$ & $6.7(1.9)$ & $7.0(2.0)$ & $5.2(2.2)$ & $5.7(2.4)$ & Physio $<$ spine centre $<$ others \\
\hline $\begin{array}{l}\text { Leg pain, mean } \\
\left(\mathrm{SD}^{\mathrm{a}}\right)\end{array}$ & $2.6(2.8)$ & $3.9(3.3)$ & $3.1(2.8)$ & $4.6(2.8)$ & All different from each other \\
\hline \multicolumn{6}{|c|}{ Activity limitation $^{\mathrm{d}}(0-100 \text { scale })^{\mathrm{c}}$} \\
\hline Mean $\left(\mathrm{SD}^{\mathrm{a}}\right)$ & $51.0(24.1)$ & $58.6(23.7)$ & $55.4(23.8)$ & $59.5(22.7)$ & Chiro $<$ GP and spine centre \\
\hline \multicolumn{6}{|c|}{ STarT Back Tool risk subgroup } \\
\hline Low-risk & $215(51.7 \%)$ & $93(35.1 \%)$ & $65(32.5 \%)$ & $273(28.0 \%)$ & \multirow{3}{*}{$\begin{array}{l}\text { All differ from each other, except } \\
\text { GP and physio }\end{array}$} \\
\hline Medium-risk & $160(38.5 \%)$ & $96(36.2 \%)$ & $85(42.5 \%)$ & $312(32.0 \%)$ & \\
\hline High-risk & $41(9.9 \%)$ & $76(28.7 \%)$ & $50(25.0 \%)$ & $389(40.0 \%)$ & \\
\hline
\end{tabular}

a Standard deviation

b Numeric rating scale $(0-10)$

${ }^{c}$ High scores are worse

${ }^{\mathrm{d}}$ Roland Morris Disability Questionnaire

e Tests for differences between subgroups were ANOVA with Scheffe post hoc comparisons or Kruskal-Wallis procedures, depending on data type and distribution $(p<0.05)$ 